\title{
Linkage analysis of five pedigrees affected with typical autosomal dominant retinitis pigmentosa
}

\author{
L LEIGH FIELD* $\dagger$, JOHN R HECKENLIVELY + , ROBERT S SPARKES §, \\ CHARLES A GARCIAף, CLYDE FARSONP, DON ZEDALIS**, \\ MARYELLEN C SPARKES $\S$, MICHOL CRIST $\S$, SUSAN TIDEMAN $\dagger$, AND \\ M ANNE SPENCE* $\dagger \|$
}

From the *Department of Psychiatry, $\uparrow$ Mental Retardation Research Center, $\ddagger$ Department of Ophthalmology, $\S$ Division of Medical Genetics, Departments of Psychiatry, Medicine, and Pediatrics, IDepartment of Biomathematics, UCLA, Los Angeles, California; \Department of Ophthalmology, University of Texas, Houston, Texas; ${ }^{\mathrm{P}}$ US Public Health Service, Tuba City, Arizona; and **Department of Ophthalmology, University of Kentucky, Lexington, Kentucky, USA.

SUMMARY Five pedigrees (including an expanded version of a previously reported pedigree) exhibiting typical autosomal dominant retinitis pigmentosa were analysed for linkage of RP to 29 genetic markers. No significant lod scores resulted. The largest lod score is $+1 \cdot 51$ and suggests linkage between $\mathrm{RP}$ and $\mathrm{Rh}$ blood group at an estimated recombination fraction of $20 \%$ in males and $40 \%$ in females. Further studies are needed to confirm or refute this suggested linkage.

Retinitis pigmentosa (RP) is the generic name given to a group of retinopathies sharing similar clinical manifestations but having a variety of aetiologies. The disease may be inherited as a simple Mendelian trait with autosomal dominant, autosomal recessive, or $\mathrm{X}$ linked transmission. It can also occur sporadically or as a component of several syndromes. ${ }^{12}$

We have previously reported the results of linkage analysis on a large pedigree (RP01) with typical autosomal dominant RP. ${ }^{\mathbf{3}}$ Although some of the findings were modified when the computer analysis was revised, ${ }^{4}$ the lod score for $\mathrm{RP}$ with $\mathrm{Rh}$ blood group was unaltered and suggested linkage (maximum $\mathrm{Z}=+2.50$ at $10 \%$ recombination). It therefore appeared worthwhile to extend the linkage analysis by collecting data from additional members of RP01 as well as from other pedigrees exhibiting typical autosomal dominant RP. We present the results of this effort here.

\section{Materials and methods}

The original study of $\mathrm{RP}^{3}{ }^{3}$ consisted of 54 people examined for genetic markers. Another 35 members have now been typed and one child in the original analysis whose RP status was uncertain has been deleted from this analysis. In addition to these 88 subjects, the linkage analysis included 40 people Received for publication 13 November 1981 not available for blood studies but necessary in the pedigree as the parent of, or link between, typed members. The pedigree is shown (figs 1,2 ) as it was analysed, in two parts called RP01B and RP01C. The affected subjects of the earliest generations are second cousins once removed. Members of the second pedigree, RP01A (fig 3), live in the same rural neighbourhood as RP01B and C, but no genealogical connection could be found. Five of six RP01A members were typed. In the remaining three pedigrees (figs $4,5,6$ ), the number of people studied for genetic markers were: RP02, 15 of 19; RP05, 47 of

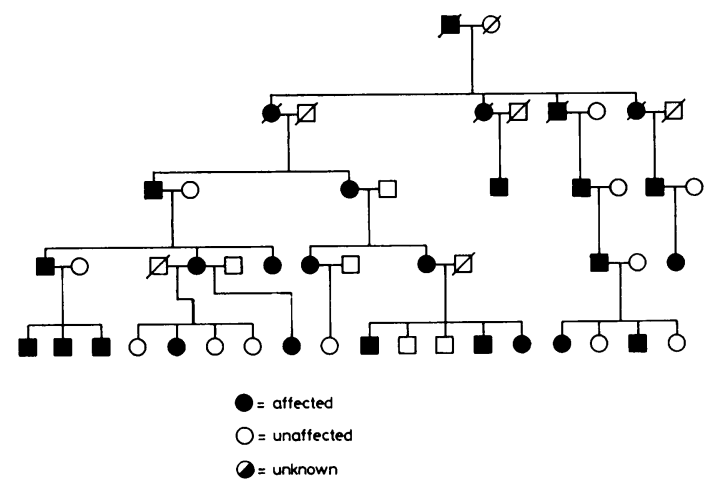

FIG 1 Kindred RPOIB 


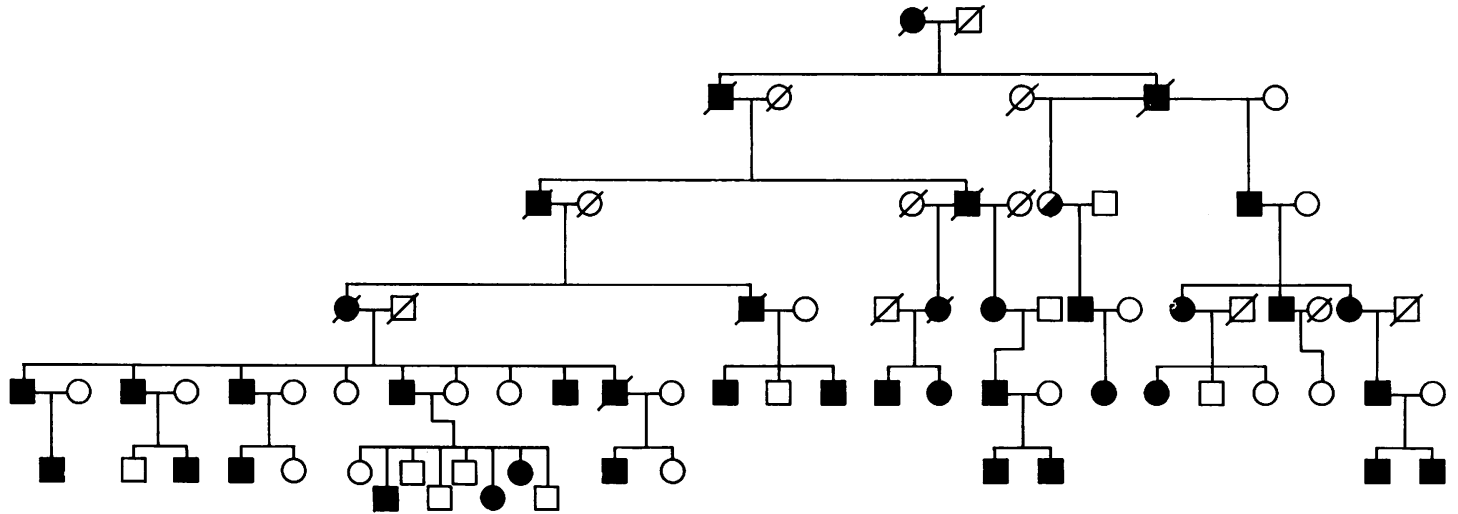

FIG 2 Kindred RPOIC

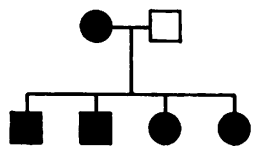

FIG 3 Kindred RP01A

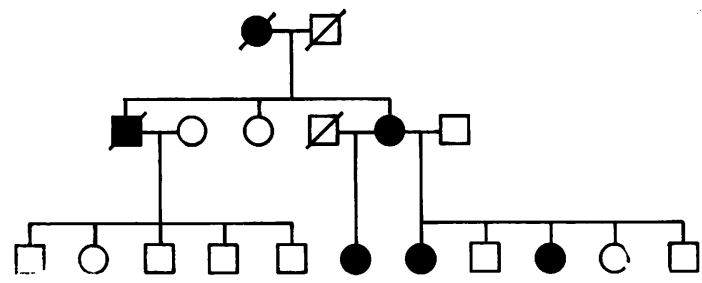

55; and RP06, 68 of 77. Thus, the analysis included 285 people, 223 of whom were typed for genetic markers.

All pedigrees are segregating typical autosomal dominant RP, which is characterised by chronic progressive degeneration of the retinal neuroepithelium (especially the rods), widespread pigmentary changes including 'bone spicule'pigment deposits, and optic nerve atrophy. The common symptoms are night blindness and gradual reduction of the peripheral visual field, which may terminate in the loss of central vision. We use the term 'typical' to distinguish this form from autosomal dominant cone-rod dystrophy, which, unlike 'typical' RP, exhibits early involvement of the cones. RP status of all members was determined by medical history and (for those whose blood was taken) also by direct examination.

Phenotypes were determined for PTC tasting

FIG 4 Kindred RPO2

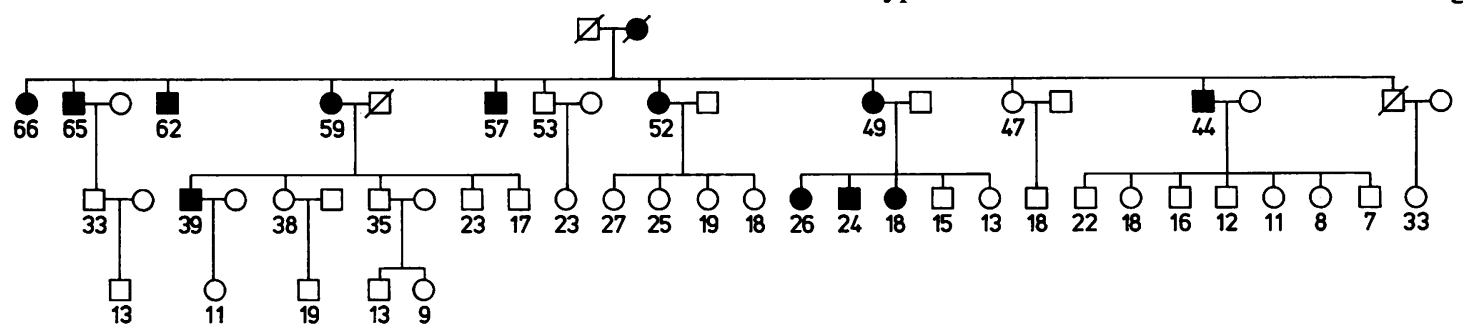

FIG 5 Kindred RPO5 with ages of affected and at risk members

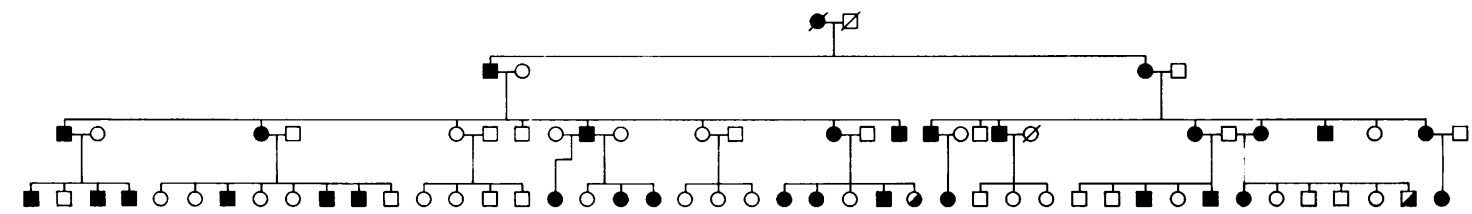

FIS 6 Kindred RP06 
ability and 28 blood markers (ABO, Le, Rh, MNSs, Fy, Lu, K, Jk, P, Ak 1 , PGD, ADA, PGM 1 , PGP, ACP $_{1}$, GALT, GPT, EsD, GLO ${ }_{1}$, HPA, TF, GC, $\left.\mathrm{CHE}_{1}, \mathrm{CHE}_{2}, \mathrm{AMY}_{2}, \mathrm{BF}, \mathrm{PGM}_{2}, \mathrm{GOT}_{1}\right)^{*}$ using standard laboratory procedures. The programme LIPED $^{5}$ was used to calculate lod scores at 36 combinations of $\theta_{\text {male }}, \theta_{\text {female }}$ (recombination fraction $\theta=0.01,0.05,0.10,0.20,0.30$, and 0.40 ) for the 16 to 23 markers that were informative in the five pedigrees. Lod scores were then summed across pedigrees. The Rhesus blood group locus $(\mathrm{Rh})$ was analysed assuming the presence of the four most common alleles: $R_{1}(C D e), r(c d e), R_{2}(c D E)$, and $R_{0}(c D e)$. The distribution of $R h$ antigens in each pedigree did not indicate the presence of other alleles.

When the genotype of a person marrying into the pedigree is not known exactly and cannot be inferred from progeny, LIPED computes probabilities of possible parental genotypes from the population gene frequencies supplied by the user. All pedigrees were analysed using gene frequencies estimated from large samples of people of European origin, except RP06 which is Navajo Indian. For the analysis of this

* Gene symbols are those used by the Human Gene Mapping Conference, Edinburgh, 1979 (Cytogenet Cell Genet 1979:25). pedigree, we located Navajo gene frequency estimates for nine of the informative markers (including Rh), south-west Amerindian frequencies for another four, and were forced to use Caucasian frequencies for the remaining ten informative markers.

Examination of the age of onset range within each pedigree revealed that RP05 and RP06 had members unaffected but at risk. Since the onset range for RP06 was narrow (age 6 to 8), two members less than 8 years old were simply called 'unknown' for RP phenotype. On the other hand, RP05 had a wide onset range (age 10 to 25 ) and there were 20 unaffected members less than 25 years old (see figure 5). For this pedigree, we applied a sub-routine to LIPED developed by Hodge et $a l^{6}$ which adjusts the penetrance of the RP gene according to age.

\section{Results}

For simplicity, we report lod scores calculated at $\theta$ male $=\theta$ female (table 1$)$. The maximum calculated score is marked with an asterisk or is given in the last column if it occurred at $\theta_{\mathrm{m}} \neq \theta_{\mathrm{r}}$. No statistically significant lod score (lod $\geqslant 3.0$ ) was produced, the largest score being +1.51 for RP-Rh at $\theta_{\mathrm{m}}=0.20$,

TABLE 1 Lod scores between $R P$ and genetic markers $\left(\theta_{m}=\theta_{\mathrm{q}}\right) \dagger$

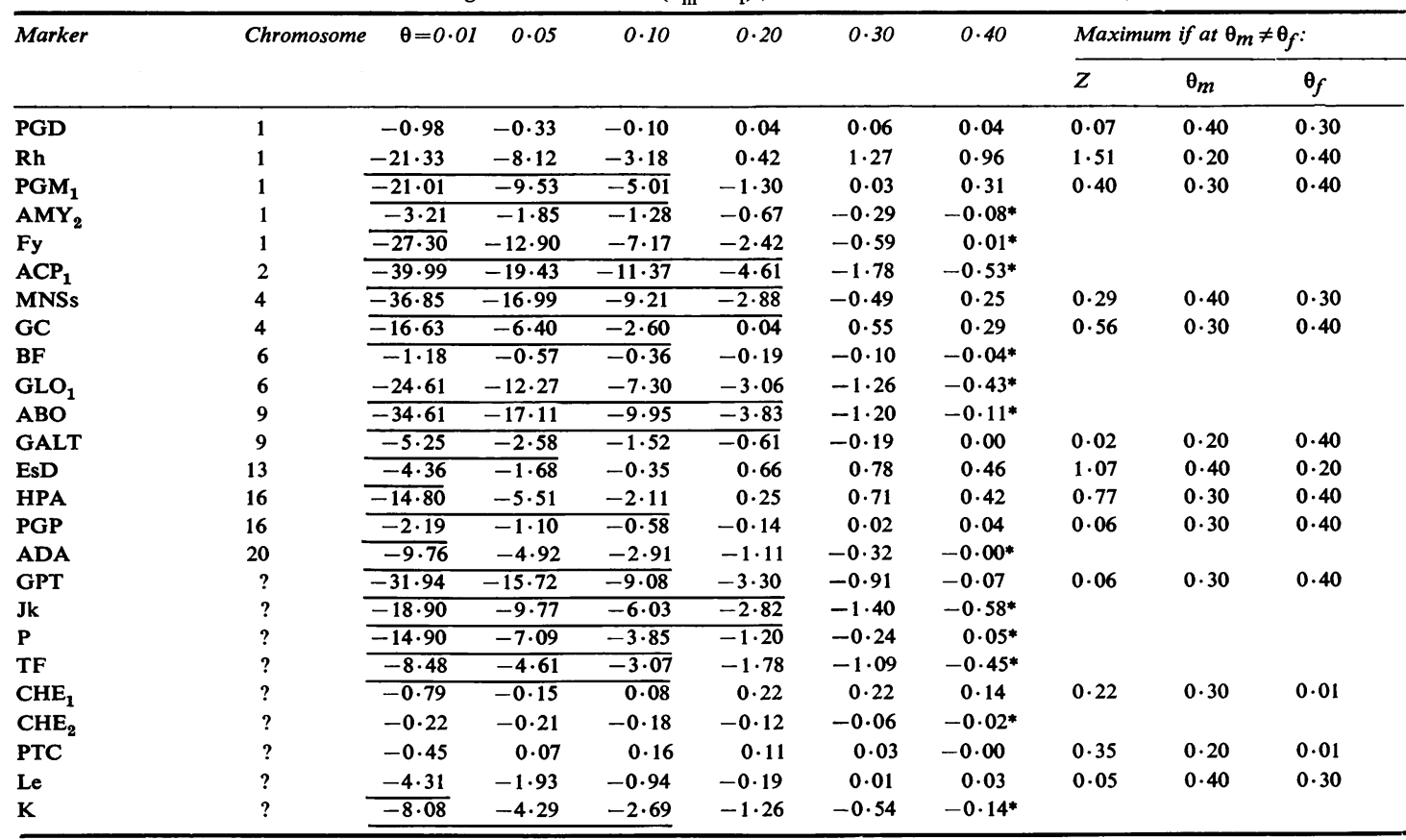

The maximum calculated lod score is marked with an asterisk, unless it occurred at $\theta_{\mathrm{m}} \neq \theta_{\rho}$, in which case it is given in the last column of the table. Scores $\leqslant-2 \cdot 0$ (linkage excluded at that $\theta$ ) are underlined. All lod scores for $\mathbf{A K}_{1}$ and GOT $_{1}$ were less than absolute value $0 \cdot 01$. 
TABLE 2 Lod scores with $R h$ and nearby chromosome 1 markers for the pedigrees separately $\left(\theta_{\mathrm{m}}=\theta\right) \dagger$

\begin{tabular}{|c|c|c|c|c|c|c|c|c|c|}
\hline & \multirow[t]{2}{*}{$\theta=0.01$} & \multirow[t]{2}{*}{0.05} & \multirow[t]{2}{*}{$0 \cdot 10$} & \multirow[t]{2}{*}{0.20} & \multirow[t]{2}{*}{$0 \cdot 30$} & \multirow[t]{2}{*}{0.40} & \multicolumn{3}{|c|}{ Maximum if at $\theta_{\mathrm{m}} \neq \theta_{f}$} \\
\hline & & & & & & & $Z$ & $\theta_{m}$ & $\theta_{f}$ \\
\hline $\begin{array}{r}\text { RP-PGD } \\
\text { RP06 }\end{array}$ & -0.98 & -0.33 & $-0 \cdot 10$ & 0.04 & 0.06 & 0.04 & 0.07 & 0.40 & $0 \cdot 30$ \\
\hline $\begin{array}{l}\text { RP-Rh } \\
\begin{array}{l}\text { RP01 B + C } \\
\text { RP02 } \\
\text { RP05 } \\
\text { RP06 }\end{array}\end{array}$ & $\begin{array}{c}-7.07 \\
0.31 * \\
-5 \cdot 85 \\
-8.71\end{array}$ & $\begin{array}{r}-1 \cdot 90 \\
0.28 \\
-3.08 \\
-3.43\end{array}$ & $\begin{array}{r}-0.90 \\
0.25 \\
-1.92 \\
-1.42\end{array}$ & $\begin{array}{c}0.97^{*} \\
0.17 \\
-0.84 \\
0.12\end{array}$ & $\begin{array}{r}0.95 \\
0.09 \\
-0.33 \\
0.56\end{array}$ & $\begin{array}{c}0.54 \\
0.03 \\
-0.08^{*} \\
0.47\end{array}$ & 0.92 & $0 \cdot 10$ & 0.40 \\
\hline $\begin{array}{c}\text { RP-PGM } \\
\text { RP01 B +C } \\
\text { RP01A } \\
\text { RP02 } \\
\text { RP05 } \\
\text { RP06 }\end{array}$ & $\begin{array}{r}-12 \cdot 98 \\
-0.57 \\
-0.51 \\
-2 \cdot 47 \\
-4 \cdot 47\end{array}$ & $\begin{array}{r}-5 \cdot 58 \\
-0 \cdot 56 \\
0 \cdot 10 \\
-1 \cdot 14 \\
-2 \cdot 35\end{array}$ & $\begin{array}{r}-2 \cdot 72 \\
-0.47 \\
0.28 \\
-1 \cdot 64 \\
-1 \cdot 45\end{array}$ & $\begin{array}{l}-0.49 \\
-0.27 \\
0.31^{*} \\
-0.23 \\
-0.62\end{array}$ & $\begin{array}{r}0.25 \\
-0.11 \\
0.21 \\
-0.07 \\
-0.24\end{array}$ & $\begin{array}{c}0.33 \\
-0.03^{*} \\
0.07 \\
-0.01^{*} \\
-0.05^{*}\end{array}$ & $0 \cdot 36$ & $0 \cdot 30$ & 0.40 \\
\hline
\end{tabular}

†The maximum calculated lod score is marked with an asterisk, unless it occurred at $\theta_{m} \neq \theta_{f}$, in which case it is given in the last column of the table. Scores for uninformative pedigrees (lods $=0.00)$ are omitted.

TABLE 3 Rh data by pedigree $\dagger$

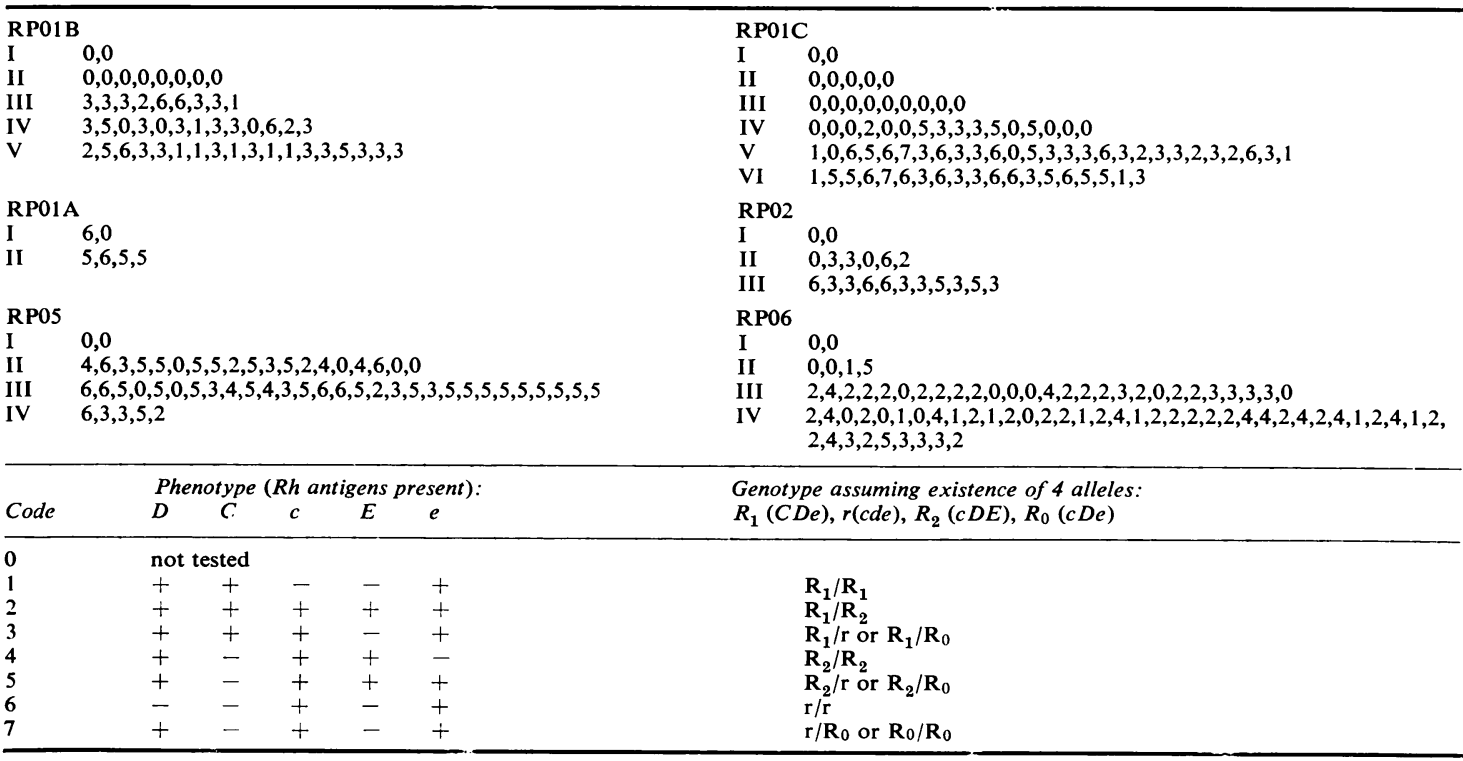

†The $\mathrm{Rh}$ phenotype codes for every subject in figs 1 to 6 are listed by generation, from left to right. The phenotype represented by each code, and the genotype interpretation used in the linkage analysis, is given at the bottom of the table.

$\theta_{\mathrm{f}}=\mathbf{0} \cdot 40$. The $\mathrm{Rh}$ locus has been mapped to the short arm of chromosome $1 .^{7}$ Scores with other chromosome 1 markers are maximal at $\theta$ values indicative of loose or no linkage. Other lod scores greater than +0.50 are: +1.07 for RP-EsD, +0.77 for RP-HPA, and +0.56 for RP-GC. Scores of $\leqslant-2 \cdot 0$ are underlined in table 1 ; they are evidence against linkage of RP to the marker at that and smaller $\theta$ values.

Table 2 presents lod scores of RP with Rh for the pedigrees separately, since it may be inappropriate to sum across pedigrees if more than one RP locus is involved. Scores for RP with chromosome 1 markers that are within mapable distance of $\mathrm{Rh}$ are also given. Table 3 gives the $\mathrm{Rh}$ phenotype data for all pedigrees.

\section{Discussion}

The lod score of +1.51 suggests loose linkage of $\mathrm{RP}$ with the $\mathrm{Rh}$ blood group locus. In summing lod scores across pedigrees, we are assuming that the same RP locus is involved in all cases. Clinically, there is no basis for predicting otherwise. Although 
the age of onset range differed between pedigrees, this may reflect differences in efforts at early diagnosis. Thus, the narrow onset range in the Navajo family could result from their being regularly examined by an ophthalmologist. The three larger pedigrees (RP01B+C, RP05, and RP06) all had at least some members diagnosed by the age of 10 .

In the absence of biological evidence of heterogeneity, one can search for evidence of heterogeneity in the recombination fraction. Morton ${ }^{8}$ proposed a test for linkage heterogeneity such as might result from involvement of more than one disease locus: the quantity $4.605\left(\Sigma \hat{z}_{1}-\hat{Z}\right)$ approximates a $\chi^{2}$ distribution with $n-1$ degrees of freedom, where $\hat{z}_{i}$ is the maximum score at $\theta_{\mathrm{m}}=\theta_{\mathrm{f}}$ for $\mathrm{i}^{\text {th }}$ pedigree, $\hat{Z}$ is the maximum summed score at $\theta_{m}=\theta_{\ell}$ for the combined pedigrees, and $\mathrm{n}$ is the number of pedigrees. We performed this test for RP-Rh (interpolating for $\hat{Z}$ and $\hat{z}_{i}$ ) and did not find significant heterogeneity $(p=0.67)$.

If $R P$ is linked to $R h$, it would also show linkage to PGD on the pter side of $\mathrm{Rh}$ (recombination fraction $\left.\theta_{\mathrm{m}}=0 \cdot 17, \theta_{\mathrm{f}}=\mathbf{0 . 2 7}\right)^{7}$ or to $\mathrm{PGM}_{1}$ on the qter side of $R h$ (recombination fraction $\theta_{m}=0.25$, $\left.\theta_{\mathrm{f}}=\mathbf{0} \cdot 38\right) .{ }^{7}$ Our present estimate of recombination fraction for RP-Rh would place the RP locus close to the $\mathbf{P G M}_{1}$ locus if it were on the qter side. Since close linkage of RP-PGM ${ }_{1}$ has been excluded by our data, the more likely location of the RP locus is on the pter side of Rh. Our scores with PGD are not inconsistent with this location; however they provide almost no information on the matter.

Hussels-Maumenee et al $^{9}$ examined three pedigrees for linkage relations of autosomal RP to 15 markers. No lod score greater than $+\mathbf{0 . 5 0}$ was obtained. Scores with $\mathrm{Rh}$ were negative and when these are summed to our results, the lod scores at $\theta_{\mathrm{m}}=\theta_{\mathrm{p}}=$ $0 \cdot 10,0 \cdot 20,0 \cdot 30$, and 0.40 become $-6 \cdot 50,-1 \cdot 04$, +0.71 , and +0.83 , respectively. Since the maximum score we calculated for RP-Rh occurs at loose recombination fractions, a large number of informa- tive matings will be needed to bring this score to +3.0 or -2.0 and thereby establish or reject linkage.

We wish to thank $\mathrm{K} M$ Joe and $\mathrm{J}$ Chen for computer assistance and $\mathrm{D}$ Bruels for manuscript preparation. This research was supported in part by grants MCH-927 and HD-04612 from the US Public Health Service and a grant from the Retinitis Pigmentosa Foundation. We are indebted to $\mathrm{Mr}$ and Mrs Arthur Golden for their generous contribution in memory of Margaret Helen Weston.

\section{References}

1 McKusick VA. Mendelian inheritance in man. Catalogs of autosomal dominant, autosomal recessive and $X$-linked phenotypes. 5th ed. Baltimore: Johns Hopkins University Press, 1977.

2 Merin MD, Auerbach E. Review, retinitis pigmentosa. Survey Ophthalmol 1976;20:303-46.

3 Spence MA, Sparkes RS, Heckenlively JR, et al. Probable genetic linkage between autosomal dominant retinitis pigmentosa (RP) and amylase (AMY2): evidence of an RP locus on chromosome 1. Am J Hum Genet 1977;29: 397-404.

4 Spence MA, Sparkes RS, Heckenlively JR, et al. Erratum. Am J Hum Genet 1977;29:592.

5 Ott J. A computer program for linkage analysis of general human pedigrees. Am J Hum Genet 1976;28:528-9.

- Hodge SE, Morton LA, Tideman S, Kidd KK, Spence MA. Age-of-onset available for linkage analysis (LIPED). Am J Hum Genet 1979;31:761-2.

7 Cook PJL, Hamerton JL. Report of the committee on the genetic constitution of chromosome 1. Cytogenet Cell Genet 1979;25:9-20.

8 Morton NE. The detection and estimation of linkage between the genes for elliptocytosis and the $\mathrm{Rh}$ blood type. Am J Hum Genet 1956;8:80-96.

9 Hussels-Maumenee I, Pierce ER, Bias WB, Schleutermann DA. Linkage studies of typical retinitis pigmentosa and common markers. Am J Hum Genet 1975;27:505-8.

Requests for reprints to Dr L Leigh Field, Division of Medical Genetics, Neuropsychiatric Institute, 760 Westwood Plaza, University of California, Los Angeles, California 90024, USA. 